\title{
SURF and LA with RGB Vector Space Based Detection and Monitoring of Manholes with an Application to Tri- Rotor UAS Images
}

\author{
Zain Anwar Ali, Dao Bo Wang, and Muhammad Shafiq Loya
}

\begin{abstract}
The detection of manhole's in a road by using Unmanned Aerial System or Vehicle (UAS) offers significant precision, however detection of manholes in above ground level images is a difficult task, with the help of Digital Single Lens Reflex (DSLR) camera and by using an image processing technique it is quite easy to detect small objects from the aerial view. Previously many methods is used to detect the manhole's covers, and many of them is quite accurate about $90 \%$ accuracy rate. But all the method applied for the detection of a covered manhole, but no one detects if there is no manhole cover lie in a manhole frame. This manuscript we present Speeded-Up Robust Feature's (SURF) algorithm for the key point detection of the manhole cover is present or not, and by marking many key points detects the manhole. After that cross checking our algorithm accuracy by using another method Learning Automata (L.A) with color gradient vector Red, Green, Blue (RGB) for the detection of a circular shape of manhole cover and color recognition for the cover is present or not.
\end{abstract}

Index Terms-Manhole detection, speeded-up robust feature, Learning automata, RGB vector space.

\section{INTRODUCTION}

Karachi is the 2nd biggest city of the world with respect to its urban population. The most populated cities have their utility issues like electricity, gas, water and sewerage that would be solved by the municipalities or Metropolitan Corporation. In this paper our main concerned is the surveillance of the manholes in the city. According to Karachi water and sewerage board (KW\&SB). The Karachi city have 3527 union councils and the total length of sewer is about $5670 \mathrm{Km}$ and an about 250000, number of manhole's in the city.

For checking or to monitor every manhole in the city it requires a lot of people and time to do that work and also it is not possible to check every manhole in the city. Manhole inspection and maintenance is the main issue for the local metropolitan corporation those manholes is covered with a metallic made or cement grey color cover. On the other hand, if the cover is broken or removed cause by any factors it is quite dangerous for the pedestrian and moving cars. Hence a competent and reliable system is required for the

Manuscript received September 16, 2015; revised November 25, 2015.

Zain Anwar Ali and Dao Bo Wang are with College of Automation Engineering, Nanjing University of Aeronautics and Astronautics. Nanjing, China (e-mail: zainanwar86@hotmail.com).

Muhammad Shafiq is with Electronic Engineering Department of Sir Syed University of Engineering and Technology, Karachi, Pakistan. continuous detecting and monitoring of the manhole.

For this purpose, the small Unmanned Aerial Vehicle or System (UAV or UAS) with image processing algorithm use for the continuous and precise monitoring of urban areas. UAV are small, ecological and silent aerial platforms which allow collecting images from a very low altitude in dangerous environments, and over long periods of time. Previously in [1] an innovative and simplest method for the obstacle detection and UAV is equipped with low cost infrared and ultrasonic finders also in [2] a persistent surveillance of the sensitive area's environmental monitoring search and rescue missions and many other applications.

However in, the above references show that how to detect the manholes previously. The authors in [3] only able to detect the cover of manholes using a geometrical filter on very high resolution aerial and satellite images by using gray color object detection and the success rate is very low about $42 \%$. In [4] two techniques is applied to detect the manholes first one searches the circular pattern using the Bhattacharyya coefficient while another one uses for the machine learning to construct a model of manhole cover the accuracy rate of this algorithm is also not good only $40 \%$ covers were detected. An automated detection of a manhole and sewage covers from LIDAR point cloud based on a marked point process of disks and rectangles is discussed in [5]. In which dissimilar from the current object detection algorithm base on the Bayesian paradigm. That method provides a maximum excellence about $92.67 \%$ from all previous methods it shows a very high precision rate.

This current algorithm is not so much suitable for detecting manhole and sewer wells cover in very high resolution optical images due to their intricate surface and intensity distributions. A parametric intensity model in [6] which defines a spherical formed manhole cover model with several parameters for correspondingly representing the radii and intensity distributions. Localization of manhole covers was achieved by directly fitting this model to the observed image intensities using Mobile Laser Scanning (MLS). Via quantitative assessments on the detection results, the projected method achieved an average correctness is about $91 \%$.

This paper presents a manhole detection application using small UAV along with two different algorithm is applied for the testing. In [7] Bay et al. presents a Scale Invariant feature recognizer called as Speeded-Up Robust Feature (SURF) for quickly computing and comparing of image features. SURF is an extended version of Scale Invariant Feature Transform (SIFT). The SIFT method applied for 
key spot description, recognition and matching via 2-D Haar-Wavelet responses and it is invariant regarding smooth scaling and point of reference. By using Difference of Gaussian filters (DOG), SIFT is not fast for online or real world applications. SURF is more robust faster and efficient for real time applications, has become the most widely used in the field of vision, science and computing. We use SURF algorithm for comparing the manhole images and recognition of manhole cover by comparing predefined images with the real images. After the competent results we apply another method for the validity of our proposed results by using Learning Automata to detect the circles of the manholes in the road and RGB vector space to specify the detection of manhole cover is present or broken.

The manuscript is divided in to five main sections. Section III we propose our both algorithms firstly SURF algorithm is used to detect the manholes in images after that cross check our results by using LA with RGB vector space to detect the circular shape of manhole covers and presence of the manholes. The 3rd section defines the type of UAS system is used to fulfill our experimental requirements along with a DSLR camera. Section IV describes the experimental results and comparing of our proposed algorithms. The last section of the manuscript defines the conclusion.

\section{ALGORITHMS}

By taking the RGB images obtain by UAS above the urban street. And after that apply both algorithms to check the robustness, precision and correctness.

\section{A. Speeded-up Robust Feature}

The complete algorithm of Speeded-Up Robust Feature is defined by the following flowchart in the figure 1 .

SIFT has a benefit it requires a low amount of image information, with high speed and precision, but it will take too much time to calculate the data [8]. In [9] by comparing SURF with the SIFT method, it adopts the (DOG) for the feature point extraction while SURF uses an integral approximation as a determinant of Hessian blob recognizer, and they can work really very fast with an integral image. To find the similarity between two images of the same scenario or detection of an object in two same images is a done by using computer vision system tool. To look for similar points in an image three main steps are required.

The interest point detector, a neighborhood of interest point and interest point descriptor. For checking the similarity of two images Euclidean distance is used. The main theme is to build a better detector and best descriptor. For real time applications like in this paper we built a real time scenario for the detection of manholes cover detection the three main points (the detector, the descriptor and the matching) to fulfill our goal. Various interest point detection scheme is available but the Fast-Hessian detector gives the better results with respect to the robustness and speed. While for the descriptor point of view Haar-Wavelet responses with in the interest point neighborhood is used. In this paper we proposed the method of integral images that allows the fastest execution of a box type convolution filter. By considering $I$ be the practiced digital image defined by the pixel's grid $\omega=[0, n-1] \times[0, m-1]$, where $\mathrm{n}$ and $\mathrm{m}$ are positive integers.

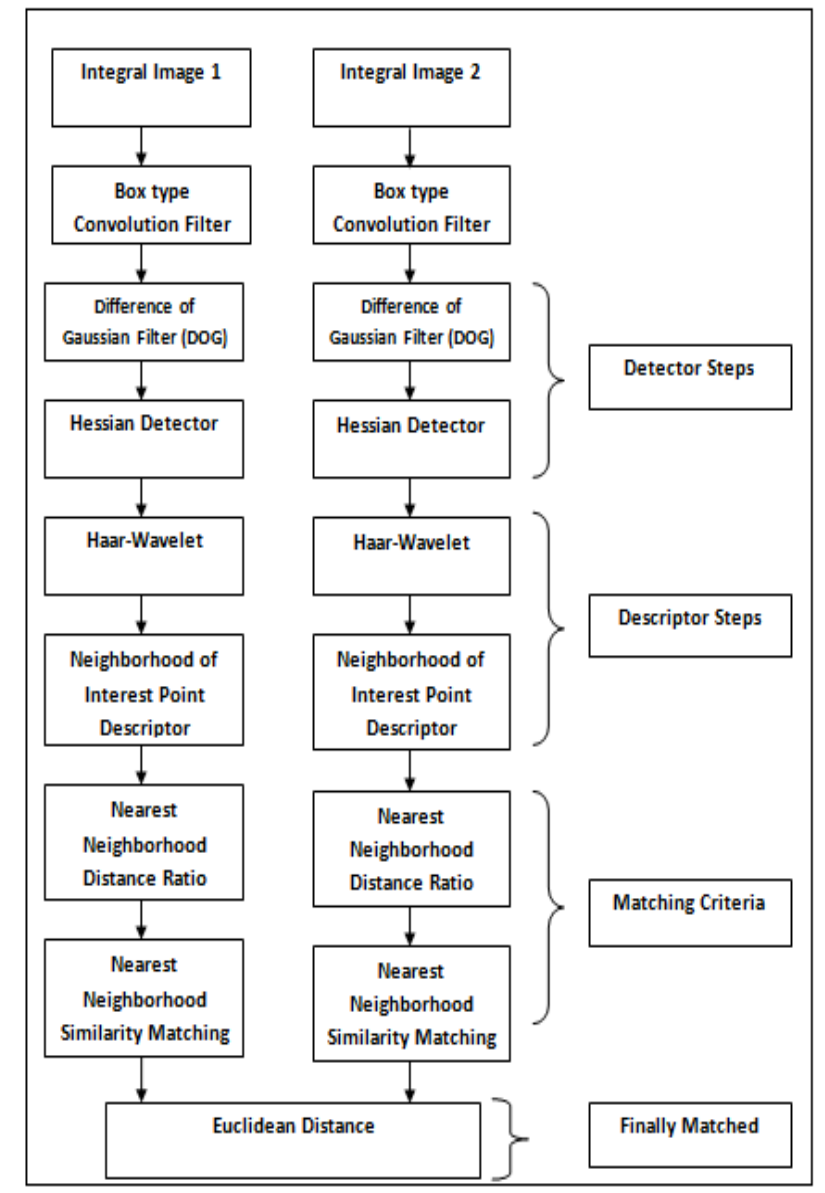

Fig. 1. The complete algorithm of speeded-up robust feature (SURF).

By taking the gray scale image having range $[0,255]$ the straightforward method to reached color adaptation, Such as black and white color tuning. The integral image $I_{m}(p)$ at a position $p=(x, y)$ by representing the sum of all pixels in the image I of a rectangular region form by point $p$.

The integral image of I for $(x, y) \in \omega$ is defined as.

$$
I_{m}(p)=\sum_{0 \leq i \leq x} \sum_{0 \leq j \leq y} I(i, j)
$$

In $I_{m}$ calculations the sum of intensities is calculated by rectangular area and independent of its size. By using an integral image filter of any size is exactly applied with the same speed.

\section{A. The Detector}

For detection, Hessian matrix is used because of its better speed, accuracy and time performance. By using Hessian determinant at a position or point $p=(x, y)$ in the imageI. The determinant is defined as $H(p, \sigma)$ in p by using a scale of $\sigma$.

$[\therefore \sigma=$ The scale at which points were detected]

$$
H(p, \sigma)=\left[\begin{array}{ll}
L_{x x}(p, \sigma) & L_{x y}(p, \sigma) \\
L_{y x}(p, \sigma) & L_{y y}(p, \sigma)
\end{array}\right]
$$

where $L$ is the convolution of the image with the second derivative of the Gaussian. In order to speed up the 
computation in SIFT algorithm Therefore the convolution is defined as the Gaussian second order derivative.

$$
L_{x x}(p, \sigma)=I(p) \times \frac{\partial}{\partial p^{2}} \times g(p)
$$

And similarly for $L_{y x}(p, \sigma), L_{y y}(p, \sigma)$ and approximately $L_{x x}, L_{x y}, L_{y x}$ and $L_{y y}$ by using a box filter, can be computed very fast using integral images.

$$
\text { SURF: } H_{\text {Approx }}^{\text {SURF }}=\left[\begin{array}{ll}
\widehat{L_{x x}} & \widehat{L_{x y}} \\
\widehat{L_{y x}} & \widehat{L_{y y}}
\end{array}\right]
$$

For selecting the location and scale of interest points SURF uses the determinant of $\mathrm{H}_{\text {Approx }}^{\text {SURF }}$ to find both, approximation using box filter.

$$
\operatorname{det}\left(H_{\text {Approx }}^{\text {SURF }}\right)=\widehat{L_{x x}} \widehat{L_{y y}}-\left(0.9 \widehat{L_{x y}}\right)^{2}
$$

The images are repetitively leveled with a Gaussian and consequently reach a superior intensity of the pyramid. In Fig. 2 (a), Fig. 2 (b) the detection method is shown.

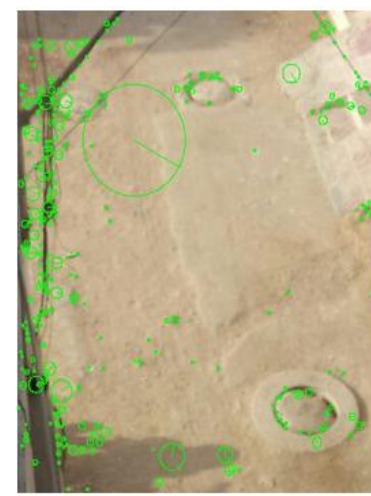

(a)

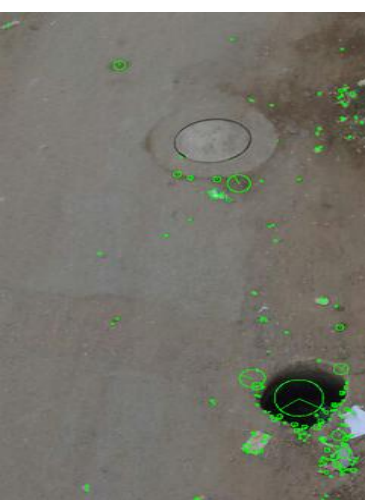

(b)
Fig. 2. (a) \& (b) Detection of a covered and open manhole using DOG in Matlab.

\section{B. The Descriptor}

Once the interest point detection is localized both in space and scale, the next steps are orientation assignment and point descriptor.

An Orientation Assignment. Initially to, calculate the $x, y$ coordinate of Haar-wavelet. A circular neighborhood having radius $6 \sigma$ around the interest point, can be computed very fast using integral images Haar-wavelet responses weighted with the Gaussian side length of wavelet is $4 \sigma$. The image is convolved with a two first order Haar wavelets, the response of the filter at a assured variety of points in the region of the orientation as a vector in a two dimensional space. A $60^{\circ}$ rotation window is applied for the summing of all the vectors within its jurisdiction and the highest valued resulting vector find out the orientation.

Point Descriptor. Firstly, by taking the square window having a size about $20 \sigma$. The total division of each sub region is divided in between $\mathrm{dx}$ and dy separately. To fetch in sequence relates to the polarity changes the intensity to extract the sum of absolute value of the entire response. The feature size of the filter is about: $4 \times 16=64$. The $\mathrm{dx}$ and dy are the wavelet responses and $|\mathrm{dx}|,|\mathrm{dy}|$ are the absolute values of the responses. The sub-region of descriptor vector is defined by.

$$
v=\left(\sum d x, \sum d y, \sum|d x|, \sum|d y|\right)
$$

The sum of all wavelet response $\mathrm{dx}$ and absolute value $|\mathrm{dx}|$ are calculated separately for points $\mathrm{dy}<0$ anddy $>0$. Vice versa for the other values of wavelet response and absolute value. Fig. 3 (a) and Fig. (b) shows the response of haar-wavelet based descriptor.

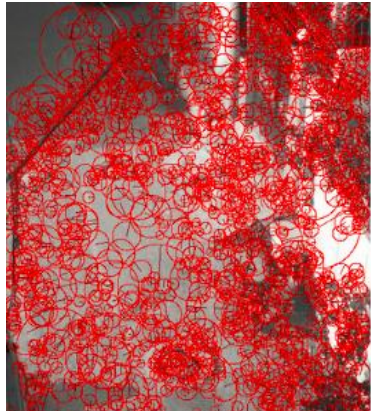

(a)

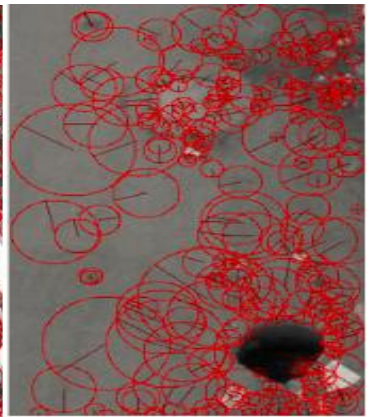

(b)
Fig. 3. (a) \& (b) Haar-Wavelet based Descriptor responses in Matlab.

\section{The Matching}

By comparing the two images $(I, J)$ which is denoted by $\left\{i_{k}\right\}$ and $\left\{j_{l}\right\}$ along their point of interest. Remembering the matrix X used for the descriptor of SURF $X \in[-1,1]$ in 64 dimensional filter feature size along with the Euclidean norm, $\|\mathrm{X}\|=1$. Euclidean distance and Nearest Neighbor Distance Ratio provides the platform for the matching of two vectors using their threshold technique. From the first image I and uncertainty descriptor $i_{k}$ by calculating the Euclidean distance $d_{k, 1}^{2}=\left\|i_{k}-j_{k}\right\|^{2}$ with all the entrant features of $j_{1}$ from the following imageJ. While from a 1 mega pixel an image approximately thousand of SURF features are extracted for it. To accelerate the comparison method initially by comparing the Laplace sign and SURF feature. The prospective similarity is discarded if the signs of both descriptor dubious similar, It means that they only take similarity distances. The nearest neighbor similarity matching is a classical model and analysis only association between particular inquiry descriptor $i_{k}$ the most like nominee represented by $j_{l 1}$. Now initially by computing the nearest neighbor $j_{l 1}$ and $j_{l 2}$ candidates from the uncertainty feature $i_{k}$, Where $\left(l_{1} \in d_{k, l}\right)$ and $\left(l_{2} \in d_{k, l}\right)$ after comparing their ratio for the fixed threshold. It can be computed as, If $d_{k, l 1} / d_{k, l 2} \leq t_{r}$. It correspondence $\left(i_{k}, j_{l 1}\right)$ is valid with a threshold level is 0.5 . SURF algorithm is three times faster than the SIFT algorithm. The matching of different manholes is shown in Fig. 4.

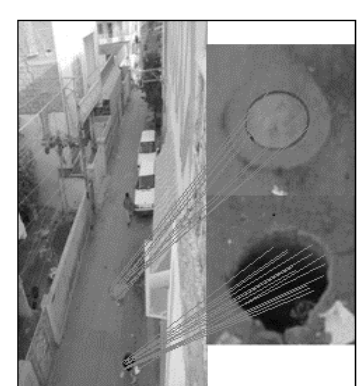

(a)

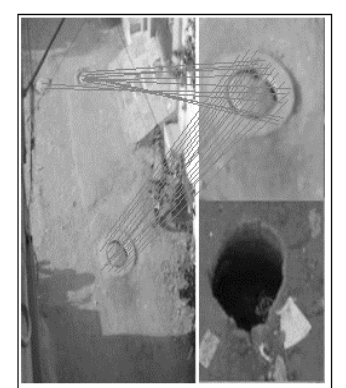

(b)
Fig. 4. (a) \& (b) Matching of images via Euclidean distance. 


\section{Cross Checking Algorithm}

\section{1) Learning automata (LA)}

Previously in [10] the LA is classified in too many variants for the suitability of different purposes. The main theme of learning structure construction not in LA is to exploit the support conventional from the environment. The linguistic sense of automaton is a self-working device or a method to facilitate a series of information in a confident mode, as a result they attain a definite target [11].

To insure the validity and effectiveness of results obtain by SURF a heuristic algorithm apply as a cross checker to assure our results Learning Automata (LA) with Red green and Blue (RGB) vector space use for the detection of multiple sewage manholes and to find the manholes is open or close. The detection of more than one manhole's in an image through only one optimization procedure LA. Previously in [12] a two-step method is used for the detection of a circle firstly detect the center of the circle by 2D Hough transform and via radius histogram check the validity of their existence. In [13] a Genetic Algorithm (GA) based approach was used to recognize the circle by using the encoding technique of the candidate circle in the image via three edge points. Both the scheme shows a good robustness and accuracy, but slow as compared to LA for multiple circular detection at a time.

For the multiple circle recognition LA based optimization with three non-collinear edge points estimates the candidate circles action. A probabilistic action $x_{i}$ from the set of different actions was performed in environmental support signal is $\beta\left(x_{i}\right)$ provided by estimation function. Linear Reward Inaction (LRI) convergence properties are as follows.

By considering an Automaton "A" with different actions called " $n$ ". Where $x_{i}$ shows the action " $i$ " set of $n$ possible actions are performed and responding to the action $x_{i}$ at time step $k$.

$$
P_{i}(k+1)=P_{i}(k)+\theta \cdot \beta\left(x_{i}\right) \cdot\left(1-P_{i}(k)\right)
$$

If $j \neq 1$ !

$$
P_{j}(k+1)=P_{j}(k)-\theta \cdot \beta\left(x_{i}\right) \cdot P_{j}(k)
$$

where $\theta$ is the learing rate and describes the value between $0<\theta<1$

and $\beta()=$.1 Indicates the maximum incentive.

and $\beta()=$.0 a null incentive is considered.

i.e. $i, j \in\{1,2,3 \ldots . . n\}$ The probability of successful action is considered to be 1 .

\section{E. Circle Detection Using LA}

\section{1) Data pre-processing}

Initially the edge detection procedure is done by selecting a canny edge detector in an image. Where $P_{t}$ the edge is vector and $\left(x_{i}, y_{i}\right)$ is the coordinates for each edge pixels $P_{i}$.

$$
P_{t}=\left\{P_{1}, P_{2}, P_{3}, \ldots P_{N T}\right\}
$$

where $N T$ is the total number of edge pixels and $i_{1}, i_{2}, i_{3}$ shows the three-point edges that must be together by assuming the circle's contour connects the points $P_{\mathrm{i} 1}, P_{i 2}, P_{i 3}$.
These points grouped in to vector "P". LA circles holding a radius within the defined limit.

Note Undersized circles are terminated by a noise having diameter lesser than 0.2

LA is based on the tracking probability evolution of each circle the candidate is also known as action. They are modified according to their actual affinity. Via the objective function which determines if the circle candidate is actually presents in the image. LA avoiding unnecessary testing of a certain image point [14].

\section{2) Representation of action}

Every circle $C_{l}$ defines by the grouping of three edge points where 1 represents the circle number. In this type of presentation an indexing is used with the edge array" $P$ ". To represent a $C_{l}$ with three edge points $\left\{P_{i}, P_{j}\right.$, and $\left.P_{k}\right\}$ and also three parameters is used to define a circle. By considering $\left(x_{o}, y_{o}\right)$ is the centre coordinates of the circle having radius $r$. The circle passes through three edges points can be written as.

$$
\left(x-x_{o}\right)^{2}+\left(y-y_{o}\right)^{2}=r^{2}
$$

The centre coordinates $\left(\mathrm{x}_{0}, \mathrm{y}_{0}\right)$ is calculated by.

$$
\begin{aligned}
& x_{o}=\operatorname{det}(A) / 4\left(\left(x_{j}-x_{i}\right)\left(y_{k}-y_{i}\right)-\left(x_{k}-x_{i}\right)\left(y_{j}-y_{i}\right)\right) \\
& y_{o}=\operatorname{det}(B) / 4\left(\left(x_{j}-x_{i}\right)\left(y_{k}-y_{i}\right)-\left(x_{k}-x_{i}\right)\left(y_{j}-y_{i}\right)\right)
\end{aligned}
$$

The determinant of matrix $A \& B$ is defined are as follows.

$$
\begin{aligned}
A & =\left[\begin{array}{ll}
x_{j}^{2}+y_{j}^{2}-\left(x_{i}^{2}+y_{i}^{2}\right) & 2\left(y_{j}-y_{i}\right) \\
x_{k}^{2}+y_{k}^{2}-\left(x_{i}^{2}+y_{i}^{2}\right) & 2\left(y_{k}-y_{i}\right)
\end{array}\right] \\
B & =\left[\begin{array}{ll}
2\left(x_{j}-x_{i}\right) & x_{j}^{2}+y_{j}^{2}-\left(x_{i}^{2}+y_{i}^{2}\right) \\
2\left(x_{k}-x_{i}\right) & x_{k}^{2}+y_{k}^{2}-\left(x_{i}^{2}+y_{i}^{2}\right)
\end{array}\right]
\end{aligned}
$$

The radius $r$ can be defined as.

$$
r=\sqrt{\left(x_{o}-x_{d}\right)^{2}+\left(y_{o}-y_{d}\right)^{2}}
$$

where $\left(x_{d}, y_{d}\right)$ are the coordinates of any three selected points in a circle and $d \in\{i, j, k\}$.

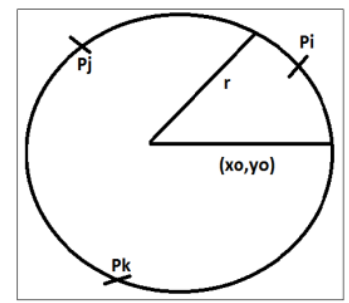

Fig. 5. $\left(P_{i}, P_{j}, P_{k}\right)$ makes a circle candidate

" $T_{m}$ " is the transformation matrix and can be represented by the shaping parameters of a circle $\left[x_{o}, y_{0}, r\right]$ and $(i, j, k)$ be the vector indexes of the transformation and can be written as.

$$
T_{m}(i, j, k)=\left[x_{o}, y_{o}, r\right]
$$

\section{3) Support signal $\beta$}

In order to calculate the error produced by the candidate solution $C_{l}$. The experimental set is defined by $S_{l}=$ $\left\{S_{1}, S_{2}, \ldots . S_{N S}\right\}$ NS are the number of points over which 
existence of an edge corresponding to $C_{l}$. The support signal $\beta_{c l}$ represents the matching error produced by the pixels of the circle candidate $C_{l}$ (action).

$$
\beta_{(c l)=\sum_{i=1}^{N S} E_{l}\left(x_{i}, y_{i}\right) / N S}
$$

where a function $E_{l}\left(x_{i}, y_{i}\right)$ verifies the pixel existence in $\left(x_{i}, y_{i}\right) \in S_{l}$ and NS is the no. of pixels lying on the corresponding to $C_{l}$. Hence the function is defined as.

$E_{l}\left(x_{i}, y_{i}\right)= \begin{cases}1 & \text { If the }\left(x_{i}, y_{i}\right) \text { is an edge point. } \\ 0 & \text { otherwise }\end{cases}$

The action is defined by two cases in the first one circle action generating similar well-known limits. The highest probability action is taken in the second step.

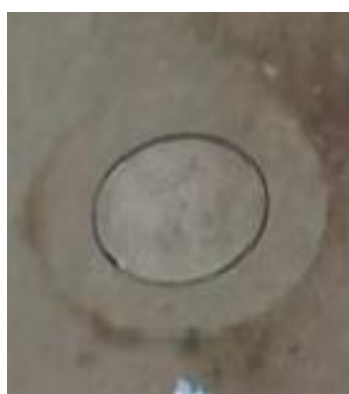

(a)

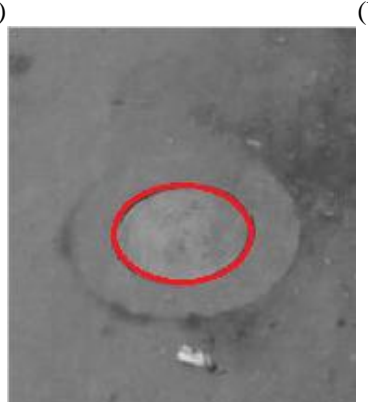

(c)

Fig. 6. (a) Input RBG-image, (b) Binary image and (c) Detection of a Circle in the gray scale image in Matlab.

\section{4) Multiple circles detection}

Previously LA is used for detecting only one circle in an image. The algorithm applied in this paper is for detecting one or more circles in an image at a same time by an optimization method. Leading with the principles of support function $(\beta)$. The location of a fixed candidate is changed via LA. The best circle candidate can fit into an actual circle within the edge-only image. The better circle means their probability is one. To detect the remaining circles in the image, the method investigating the consequential probability allocation seeking other significant local minima. To find the local minima for other circles having lower probability, the arrangement into descending order. A number of actions correspond to the same circle. A distinctiveness factor.

$$
E_{s_{d i}}=\left|x_{A}-x_{B}\right|+\left|y_{A}-y_{B}\right|+\left|r_{A}-r_{B}\right|
$$

where $\left(x_{A}, y_{A}\right)$ the central are coordinates of the circle and $r_{A}$ is the radius of the circle $C_{A}$ whereas $\left(x_{B}, y_{B}\right)$ and $r_{B}$ the corresponding parameter of the circle $C_{B}$. the difference between two circles is described by their threshold values "E $E_{S T H} "$.

$$
E_{S T H}=\left(r_{\max }-r_{\min }\right) / s
$$

Now $\left[r_{\text {max }}, r_{\text {min }}\right]$ are feasible radii ranges and $s$ is the selectivity parameter. The higher value of $s$ two similar circles would be considered as dissimilar on the other hand, the smaller s values will consider as similar circles. Once the probability distribution is achieved, the highest probability $P_{r-h i g h}$ is assigned for the first circle found. By discovering all the values, other circles are recognized by the equations (17) and (18). The method is recurring until the exploit probability goes to the minimum thresholdP $P_{\text {rth }}$. By using their threshold level lower values considered to false, later than some experiments the value of $\mathrm{P}_{\mathrm{rth}}$ is set to Pr /10high.
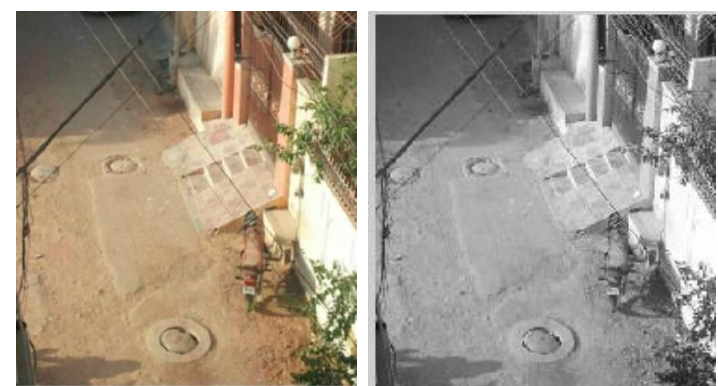

(a)

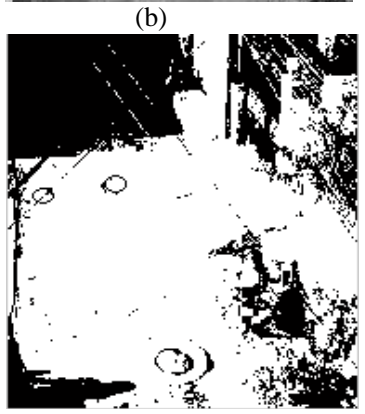

(d)

Fig. 7. (a) Input RBG-image, (b) Gray Scale Image, (c) Adjusted Image and (d) Detection of a Circle in Matlab.

After detecting or recognizing more than one manhole in an image next we use RGB vector space to check the presence of manhole cover.

\section{5) $R G B$ vector space}

In reference, [15] the RGB vector space is used with the neighborhood vector to determine the skin color of a human. To detect the human by using skin color is not an easy task. By finding each pixel of skin and threshold is used to distinguish between the neighborhoods pixels. The authors in [16] presents a method to recognize the color by using RGB color space. They use Sin of the vector angle between neighboring pixel value to point out the level of edges. The distinct Euclidean distance-based mechanism, for the detection of the edges in strength and only generate nonzero data where there are purely chromatic dissimilarity.

The RGB vector space is used to detect the gray and black color in manholes. If the manhole is covered it means gray color cover lies on it and if it is not covered it means black color, we can detect from there. We use image segmentation that divides an image in regions. A set of sample color points represents a color image in the RGB range of color interest. Let we detect a first color is gray by RGB column vector $m$. The basic objective of the division of color is to clarify each RGB pixel in an image having a 
color in that range to detect it. To achieve this contrast, we need similarity measurement via Euclidean distance.

Let $z$ denote a random point in RGB space, we can say that $z$ is equal to " $m$ " if the distance between them is less than a specified threshold, $T$ and the Euclidean distance between $\mathrm{z}$ and $\mathrm{m}$ is given by.

$$
\begin{gathered}
D(z, m)=\|z-m\| \\
=\left[(z-m)^{T}(z-m)\right]^{\frac{1}{2}} \\
=\left[\left(z_{R}-m_{R}\right)^{2}+\left(z_{G}-m_{G}\right)^{2}+\left(z_{B}-m_{B}\right)^{2}\right]^{\frac{1}{2}}
\end{gathered}
$$

where $\|$.$\| is the norm of the argument and a useful$ generalization of the preceding equation is a distance measure of the form.

$$
D(z, m)=\left[(z-m)^{T} C^{-1}(z-m)\right]^{1 / 2}
$$

where $C$ is the covariance matrix of the sample's representative of the color we wish to a segment.

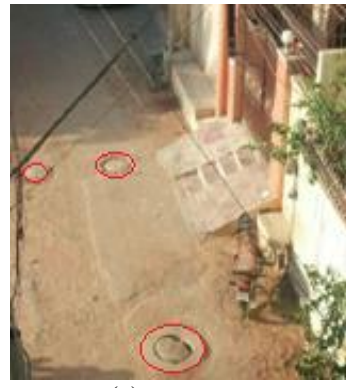

(a)

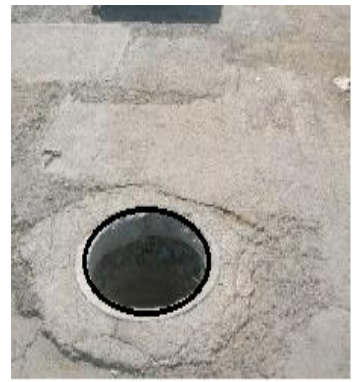

(c)

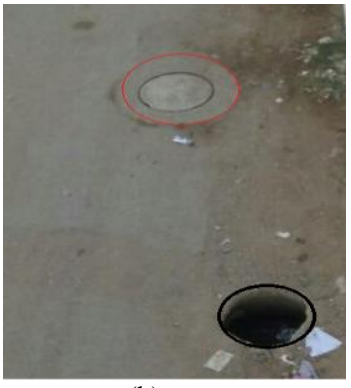

(b)

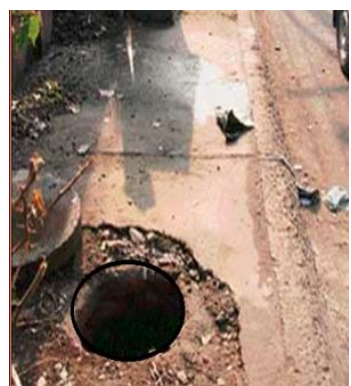

(d)
Fig. 8. (a) Detects the close manhole (b) Detects the open and close manhole (c) \& (d) Detects open manholes in Matlab.

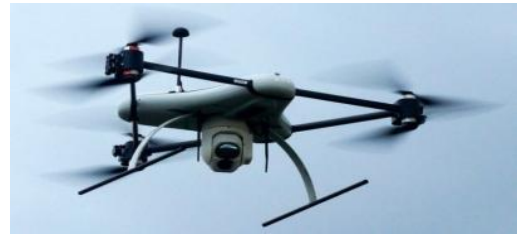

Fig. 9. The tri-rotor aerial vehicle.

\section{The UnMANNED AERIAL SySTEM}

In this experiment we use a tri-rotor aerial vehicle for the experiment [17], [18] and which was produced by Nanjing Stro-Flight Company limited (S\&mc) the professional producer of flight motion UAV and simulators. It has three rotors and in each rotor, there's a brushless motor and an electronic ardu-pilot controller is situated in the middle of the UAS. The flight controller is the main controlling unit in the system and equipped with Global Positioning System
(GPS), gyro for the direction finding, accelerometer and barometer is also equipped to maintain a particular height. A good feature digital camera is also fixed at the centrebottom of the UAS for the sake of capturing snaps at a regular interval of time see in Fig. 9.

To take the snaps from UAS initially by utilizing Google Map is downloaded in our GPS. Secondly, marked the satellite map of the particular area in which our experiment is performed shown in figure 10. After that starts the UAV at specified altitude by suing the auto pilot mode which enables to executes the flight and also taking images of the given route. Lastly those captured images computed by SURF algorithm.

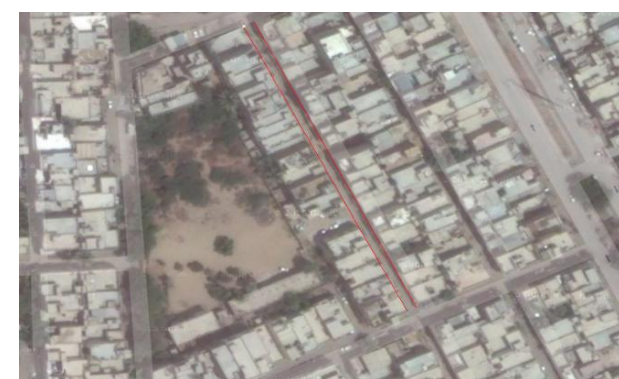

Fig. 10. Red lines set the limits of the screened area street where experiment is performed.

\section{EXPERIMENT RESULTS}

\section{A. The Description of Area}

The street where we perform our experiment is located in the North Karachi urban residential area, having latitude and longitude is approximately about 24.969 and 67.049 with the city coordinates $24^{\circ} 51^{\prime} 36^{\prime \prime} \mathrm{N} 67^{\circ} 0^{\prime} 36^{\prime \prime} \mathrm{E}$. We examined three flights on July 22nd, 2015 at 15:00, 15:30 and 16:00. Obtain the snaps over $7 \mathrm{~d}$ street \# 2 . We take that street in our experiment because in that street the number of manholes is about 19 a highest number of manholes in a small area approximately about 200 meter street is and some manholes are covered and some are not covered and for our experiment point of view we need to detect the covered and uncovered manholes and that street pays a vital role for the testing of our algorithm because the area is not too large as compared to manholes in a single street.

The images are taken at the height of $300 \mathrm{~m}$ above ground level and after that zoom in to see the street clearly as shown in Fig. 11. By using the better performance electrooptical high definition resolution (EOHDR) HD-Zoom 30 camera. With the help of this camera lens we are able to see a person face over 1000 feet height. The total number of manholes is about 19 as described earlier in which 16 are covered and three are not covered.

\section{B. Performance Estimation}

To check the performance of our entire system, by using accuracy produced $\left(A c C_{P}\right)$ accuracy rate by users $\left(A c C_{U}\right)$ and accuracy average $\left(A v_{C C}\right)$. Where $N_{m h}$ the total number of manholes is, $P_{D}$ is the accurate positive detection, $N_{D}$ is the wrong negative detection indicates that no manhole detected as a wrong point and $\mathrm{N}_{\mathrm{P}}$ is the wrong positive detection.

$$
A c c_{P}=P_{D} / P_{D}+N_{D}=P_{D} / N_{m h}
$$




$$
\begin{aligned}
& A c c_{U}=P_{D} / P_{D}+N_{P} \\
& A v_{c c}=A c c_{P}+A c c_{U} / 2
\end{aligned}
$$

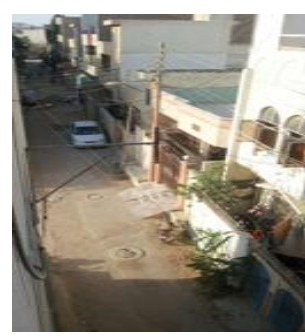

(a)

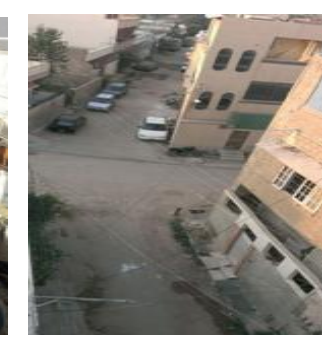

(b)

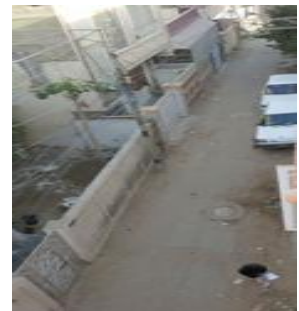

Fig. 11. (a) (b) \&(c) Zoom in images taken by UAV.

\section{Results}

We obtain different patterns that represent manholes or no manhole's respectively. And the representation of SURF key points marked with circles in the detector and descriptor step of SURF as shown in Fig. 2 and Fig. 3. And finally, the matching results is shown in Fig. 4. After that by applying LA algorithm with RGB vector space for the detection of manholes firstly we detect a single circular manhole as shown in figure 6 after that we apply algorithm for multiple circular manhole detection, which was shown in Fig. 7, we only able to detect the close manhole's and does not able to find the open manhole to resolve this issue by using RGB vector space we are able to detect the open and close manhole's easily the detection is shown in Fig. 8. The accuracy and precision of our algorithm is presented in the Table I as shown below. Our proposed algorithm works efficiently and the results shown in the Table I which verifies our algorithm cross checking.

TABLE I: DETECTION RESUlTS OF MANHOLES AT 7D STREET\#2

\begin{tabular}{lcccccc}
\hline \hline Methods & $\mathrm{M}$ & $\mathrm{PD}$ & $N_{F}$ & $A_{c c F}$ & $A_{c c U}$ & $A_{V C C}$ \\
\hline SURF & 19 & 14 & 2 & 3 & 82.3 & 84.7 \\
SURF RGB & 19 & 16 & 1 & 2 & 88.8 & 91.4 \\
LA Single & 19 & 9 & - & - & - & - \\
LA Multiple & 19 & 15 & 2 & 2 & 88 & 88 \\
LA RGB & 19 & 16 & 2 & 1 & 88.8 & 91.4 \\
\hline \hline
\end{tabular}

*Note: The values of $A_{c c F}, A_{c c U}$ and $A_{V C C}$ are in \%.

\section{CONCLUSION}

To monitor the manholes in a city it's a difficult task because it takes a lot time as well as a man power required and not possible to monitor every manhole to focus this issue in this manuscript, we propose new methods for monitoring of manholes in the urban area by using aerial images. Because advancement in technology provides us an easy reliable and time efficient procedure to do all task quickly. Initially SURF, algorithm detection scheme is applied which will follow by the descriptor and after that matching shows the validity of our first algorithm and secondly cross check our method by using LA with the color vector, space and that shows the similarity of both results it means both algorithms verify our presented method accuracy rate.

\section{ACKNOWLEDGEMENT}

Firstly we are very thankful to Mr. Muhammad Asfar who tell us this manhole issue in his living area because there is no proper system for the monitoring of manholes in Karachi. Secondly, the CEO of Nanjing Stro-Flight company who supports us to perform the experiments. Lastly, KWS\&B also provides us a useful data of the manhole as well as provides us a platform to run our experiment and monitor the manhole's effectively and hope in future KWS\&B also used our proposed method to monitor the manhole's detection.

\section{REFERENCES}

[1] N. Gageik, P. Benz, and S. Montenegro, "Obstacle detection and collision avoidance for a UAV with complementary low-cost sensors," Translations and Content Mining Are Permitted for Academic Research Only, pp. 2169-353.

[2] A. Wallar, E. Plaku, and D. A. Sofge, "Reactive motion planning for unmanned aerial surveillance of risk-sensitive areas," IEEE Transactions on Automation Science and Engineering, vol. 12, no. 3, 2015.

[3] O. Bartoli, N. Chahinian, A. Allardy, J. S. Baillyz, K. Chancibaulty, F. Rodriguezy, C. Sallesx, M. G. Tournoudx, and C. Delenne, "Manhole cover detection using a geometrical filteron very high resolution aerial and satellite images," Urban Remote Sensing Event (JURSE), 2015.

[4] J. Z. Pasquet, T. Y. Desert, O. Y. Bartoli, M. Z. X. Chaumont, C. S. G. Z. Delenne, M. Derras, and N. Y. Chahinian, "Detection of manhole covers in high-resolution aerial images of urban areas by combining two methods," Urban Remote Sensing Event (JURSE), 2015.

[5] Y. T. Yu, J. Li, H. Y. Guan, C. Wang, and J. Yu, "Automated detection of road manhole and sewer well covers from mobile LiDAR point clouds," IEEE Geoscience and Remote Sensing Letters, vol. 11 no. 9, 2014.

[6] Y. T. Yu, H. Y. Guan, and Z. Ji, "Automated detection of urban road manhole covers using mobile laser scanning data," IEEE Transactions on Intelligent Transportation Systems.

[7] H. Bay, A. Ess, T. Tuytelaars, and L. V. Gool, "Speeded-up robust features (SURF)," Comput. Vis. Image Understand, vol. 110, no. 3, pp. 346-359, Jun. 2008.

[8] W. Y. Chen, W. Z. Xie, and R. Zeng, "Method of item recognition based on SIFT and SURF," Mathematical Structures in Computer Science, vol. 24.

[9] L. Li, "Image matching algorithm based on feature-point and daisy descriptor," Journal of Multimedia, vol. 9, no. 6.

[10] M. A. L. Thathachar and P. S. Sastry, "Varieties of learning automata: An overview," IEEE Transactions on Systems, vol. 32, no. 6, 2002.

[11] B. J. Oommen, "Recent advances in learning automata systems," in Proc. 2010 2nd International Conference on Computer Engineering and Technology.

[12] D. Ioannoua, W. Hudab, and A. F. Lainec, "Circle recognition through a 2D Hough Transform and radius histogramming," Image and Vision Computing.

[13] V. A. Ramirez, C. H. G. Capulin, A. P. Garcia, and R. E. S. Yanez, "Circle detection on images using genetic algorithms," Pattern Recognition.

[14] E. Cuevas, M. P. Cisneros, V. Osuna-Enciso, D. Zaldivar, and F. Wario, "Fast algorithm for multiple-circle detection on images using learning automata," IET Image Processing, 2012.

[15] M. M. Aznaveh, H. Mirzaei†, E. Roshan†, and M. Saraee†, "A new color based method for skin detection using RGB vector space," 2008. 
[16] R. D. Dony and S. Wesolkowski, "Edge detection on color images using rgb vector angles," in Proc. the 1999 IEEE Canadian Conference on Electrical and Computer Engineering Shaw Conference Center, Edmonton, Alberta, Canada, May 9-12, 1999.

[17] Z. A. Ali, D. B. Wang, and M. Aamir, "Design a robust RST controller for stabilization of a tri-copter UAV," Pakistan Journal of Engineering Technology and Science, vol. 5, no. 1.

[18] Z. A. Ali, D. B. Wang, R. Javed, and A. Aklbar, "Modeling and controlling the dynamics of tri-rotor UAV using robust RST controller with MRAC Adaptive algorithm," International Journal of Control and Automation, vol. 9, no. 3, March 2016.

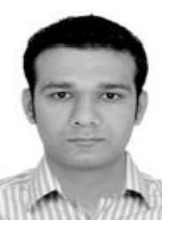

Zain Anwar Ali received his B.S. degree in electronic engineering from the Sir Syed University of Engineering and Technology, Karachi, Pakistan in 2010. And he is currently doing $\mathrm{PhD}$ in control theory and control engineering from Nanjing University of Aeronautics and Astronautics, Nanjing, China from 2015. He is working as a faculty member and researcher in the Department of Electronic Engineering, Sir Syed University of Engineering and
Technology.

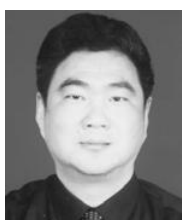

Dao Bo Wang is a professor in the College of Automation Engineering at Nanjing University of Aeronautics and Astronautics, China. He was an official designated visiting scholar at University of Bath, UK from 1987 to 1989 and senior visiting scholar at University of Waterloo, Ontario, Canada in 1999. His research interests include unmanned aerial vehicle flight control and aeronautical simulation.

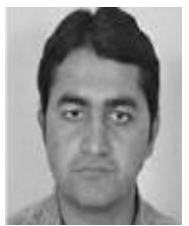

Muhammad Shafiq received his B.S. degree in electronic engineering from the Sir Syed University of Engineering and Technology, Karachi, Pakistan in 2011, and doing M.S. in telecommunication engineering from Sir Syed University of Engineering and Technology, Karachi, Pakistan. He is working as a faculty member and researcher in the Department of Electronic Engineering, Sir Syed University of Engineering and Technology. 